GA-A22989 CONF-98106\%-

\title{
COMPARISON OF L-H TRANSITION MEASUREMENTS WITH PHYSICS MODELS
}

\author{
by \\ T.N. CARLSTROM, K.H. BURRELL, R.J. GROEBNER, A.W. LEONARD, \\ T.H. OSBORNE, and D.M. THOMAS
}



DECEMBER 1998 


\section{DISCLAIMER}

This report was prepared as an account of work sponsored by an agency of the United States Government. Neither the United States Government nor any agency thereof, nor any of their employees, make any warranty, express or implied, or assumes any legal liability or responsibility for the accuracy, completeness, or usefulness of any information, apparatus, product, or process disclosed, or represents that its use would not infringe privately owned rights. Reference herein to any specific commercial product, process, or service by trade name, trademark, manufacturer, or otherwise does not necessarily constitute or imply its endorsement, recommendation, or favoring by the United States Government or any agency thereof. The views and opinions of authors expressed herein do not necessarily state or reflect those of the United States Government or any agency thereof. 


\section{DISCLAIMER}

Portions of this document may be illegible in electronic image products. Images are produced from the best available original document. 
GA-A22989

\title{
COMPARISON OF L-H TRANSITION MEASUREMENTS WITH PHYSICS MODELS
}

\author{
by \\ T.N. CARLSTROM, K.H. BURRELL, R.J. GROEBNER, A.W. LEONARD, \\ T.H. OSBORNE, and D.M. THOMAS
}

This is a preprint of a paper to be presented at the 17th International Atomic Energy Agency Fusion Energy Conference, October 19-24, 1998, Yokohama, Japan, and to be published in the Special Issue of Nuclear Fusion.

\author{
Work supported by \\ the U.S. Department of Energy \\ under Contract DE-AC03-89ER51114
}

GA PROJECT 3466

DECEMBER 1998 


\section{ABSTRACT}

A technique of fitting a modified hyperbolic tangent to the edge profiles has improved the localization of plasma edge parameters. Non-dimensional edge parameters are broadly consistent with several theories of the L-H transition that use edge gradients in their formulation of a critical threshold parameter. The ion $\nabla B$ drift direction has only a small effect on the edge plasma conditions measured near the plasma midplane but a large effect on the divertor plasma. The dramatic change of power threshold with the direction of the ion $\nabla \mathrm{B}$ drift implies that phenomena in the divertor region may be critical for the $\mathrm{L}-\mathrm{H}$ transition. 


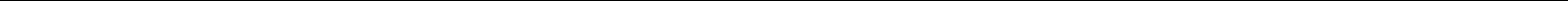




\section{INTRODUCTION}

The conditions leading to the spontaneous transition from $\mathrm{L}$-mode to $\mathrm{H}$-mode remain an important question for predicting access to $\mathrm{H}$-mode in future devices. The improved confinement in H-mode begins in a narrow region at the plasma edge and it is this area that has come under close study. Due to the presence of a separatrix, small spatial scale, neutral deuterium and impurity fluxes, and complicated geometry, this region presents challenges to study, both theoretically and experimentally. In the past few years, several models of the L-H transition have been developed that depend on non-dimensional plasma edge parameters. In this paper, we examine the characteristic of the L-mode edge region, just before the $\mathrm{L}-\mathrm{H}$ transition, and compare our findings with the predicted threshold conditions of several models.

This work represents a continuation and refinement of previous work reported in Ref. [1], where plasma edge parameters were evaluated at normalized toroidal flux surfaces, $\rho=0.90$ and 0.95, determined from the magnetic equilibrium code EFIT [2]. A conclusion from that study was the uncertainty in determining the separatrix position, $\pm 0.5 \mathrm{~cm}$, is comparable to the edge gradient scale lengths. This produced significant scatter when evaluating edge parameters at a fixed $\rho$ location. The DIII-D tokamak has excellent edge diagnostics to measure $n_{e}$ and $T_{e}$ using Thomson scattering [3] with spatial resolution of $0.75 \mathrm{~cm}$ when projected to the vessel midplane, and $T_{i}$ using charge exchange recombination [4] (CER) with spatial resolution of $0.3 \mathrm{~cm}$. The location of these measurements for a typical single-null diverted plasma is shown in Fig. 1. Typical L-mode and $H$-mode edge profiles of $n_{e}, T_{e}$ and $T_{i}$ are shown in Fig. 2, along with the separatrix position. Uncertainty in the position of the separatrix by $1 \mathrm{~cm}$ would lead to significant change in the evaluation of the edge parameters.

A technique of fitting a modified hyperbolic tangent to the edge profiles themselves has eliminated the scatter caused by the flux surface reconstruction and has improved the localization of the plasma edge [5]. A fit to a typical L-mode density profile is shown in Fig. 3. This technique allows the profile to be described with five fitting parameters that are recorded in a database. The electron density profile has the most pronounced edge gradient and is used to localize the position of the plasma edge. Figure 4 shows a comparison of the position of the density symmetry point and the density knee to the magnetically determined separatrix position for a wide range of plasmas. The density symmetry point and knee are approximately $1 \mathrm{~cm}$ and $2 \mathrm{~cm}$, respectively, inside the separatrix when mapped to the vessel midplane. The data scatter relative to the separatrix position is about $1 \mathrm{~cm}$.

Using this technique, we have determined that the position of the maximum edge density gradient (the symmetry point) remains relatively constant across the L-H transition. Figure 5 
shows the location of the electron density symmetry point measured $20-30 \mathrm{~ms}$ before the L-H transition compared with $20-30 \mathrm{~ms}$ after the transition. Although there is still some data scatter, this location remains relatively constant. Since this is the location where the confinement improvement is greatest, it is a good location to evaluate the local edge conditions relevant to the formation of the edge transport barrier in $\mathrm{H}$-mode.

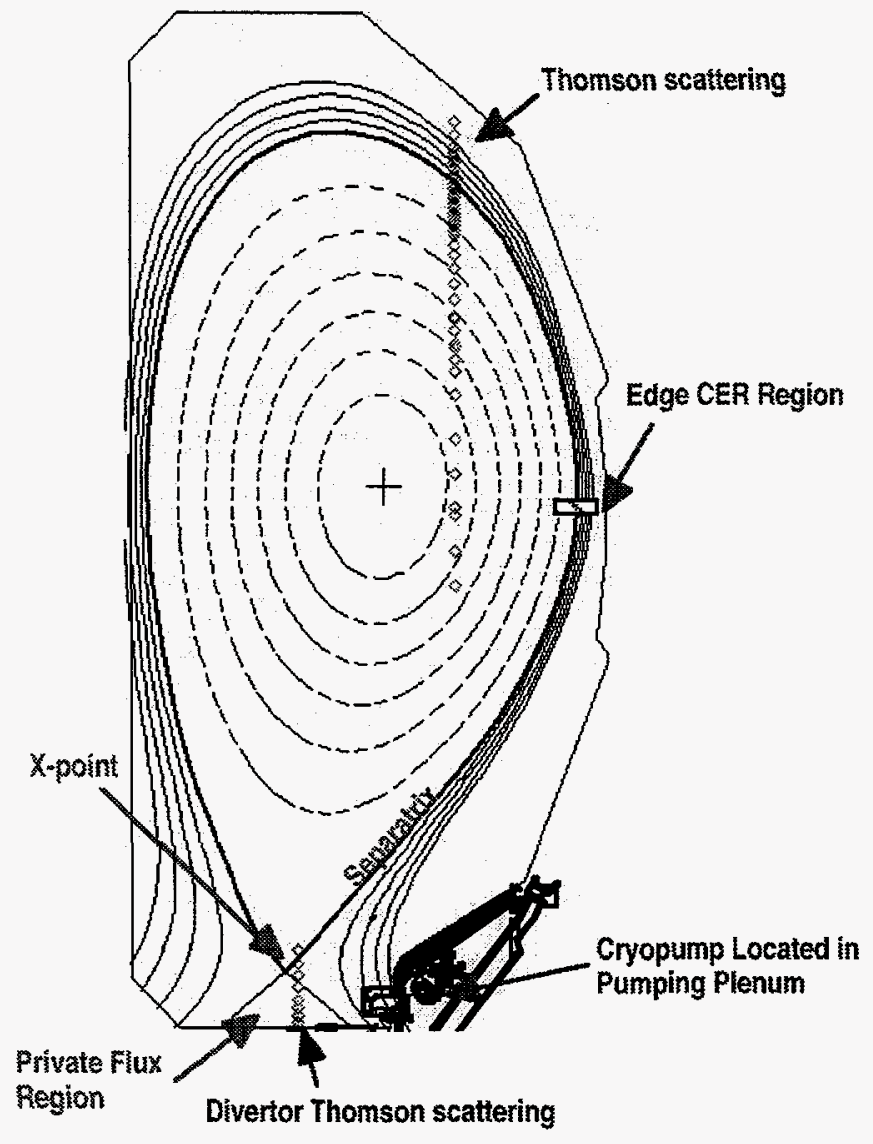

FIG. 1. A cross section of the DIII-D vacuum vessel with a flux contour plot of a typical lower singlenull diverted plasma configuration is shown along with the locations of the Thomson scattering and CER measurements. 

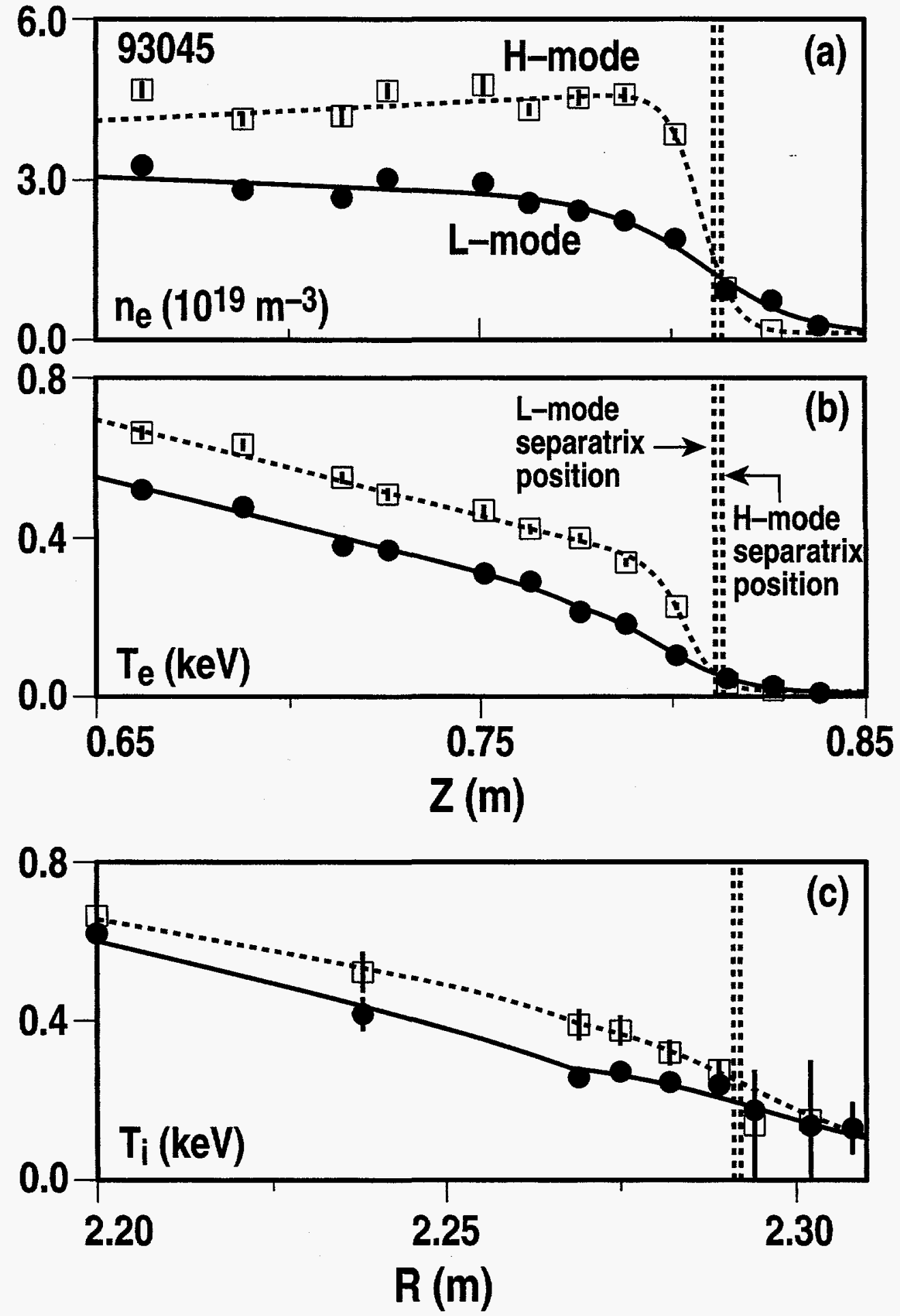

FIG. 2. Typical edge profiles of (a) $n_{e}$, (b) $T_{e}$, and (c) $T_{i}$ for $L$-mode (dots) and $H$-mode (squares). The curves are modified hyperbolic tangent fits to the data. The vertical lines show the separatrix position. 


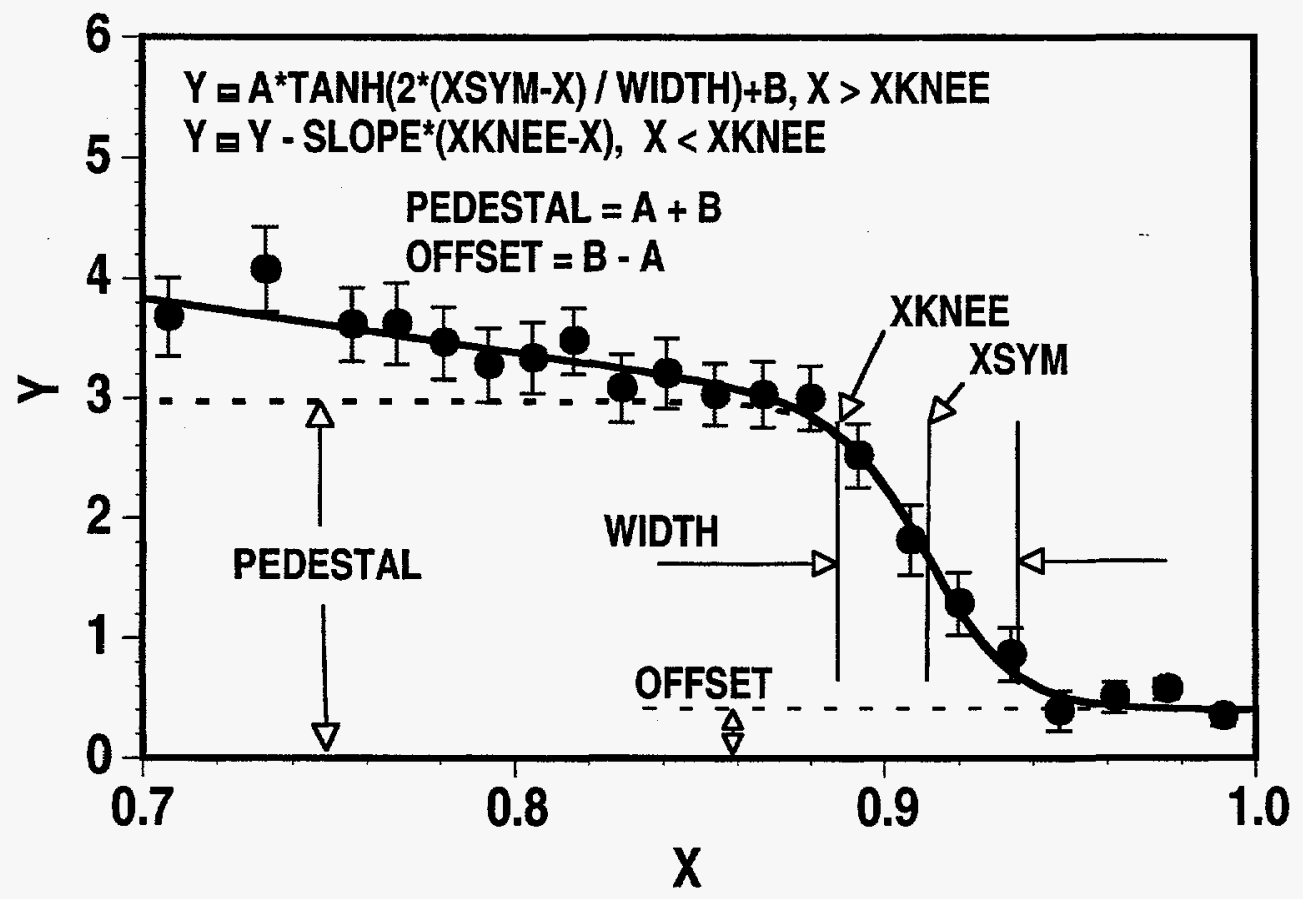

FIG. 3. Modified hyperbolic tangent fit to a typical L-mode edge $n_{e}$ profile showing the location of the symmetry point, xsym, and the profile knee, xknee.

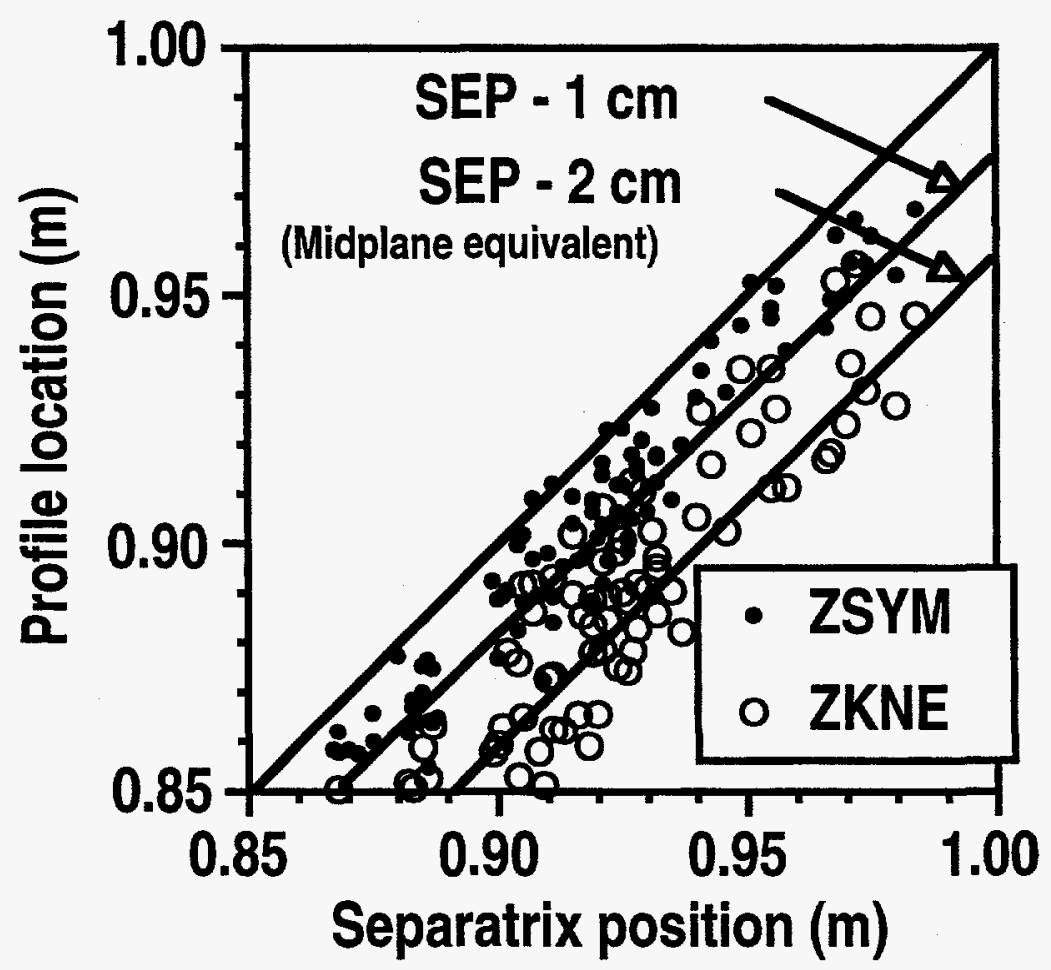

FIG. 4. Location of the $n_{e}$ symmetry point, zsym, and the profile knee, zkne, determined from the modified hyperbolic tangent fit compared with the separatrix position determined from a magnetic flux reconstruction using EFIT for a wide range of $L$-mode discharges. Also shown is the location 1 and $2 \mathrm{~cm}$ inside the separatrix when measured at the outer midplane. 




FIG. 5. Location of the $n_{e}$ symmetry point, zsym, measured just before (20-30 ms) the L-H transition compared to the location just after (20-30 ms) the transition. The location of the symmetry point remains relatively constant across the transition. 


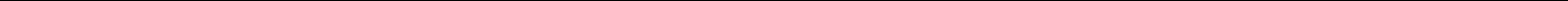




\section{LOCAL EDGE PARAMETERS}

A database of edge parameters has been assembled, covering a wide range of plasma parameters: $1.1<\mathrm{B}_{\mathrm{T}}(\mathrm{T})<2.1,1.0<\mathrm{I}_{\mathrm{P}}(\mathrm{MA})<2.0,1.2<\mathrm{n}_{\mathrm{e}}\left(10^{19} \mathrm{~m}^{-3}\right)<4.0,1.0<\mathrm{P}_{\mathrm{TH}}(\mathrm{MW})<$ 4.0. For this study, only single-null diverted deuterium plasmas with the ion $\nabla B$ drift toward the $\mathrm{X}$-point are included. In addition to edge measurements made just before (10-20 ms) the L-H transition, measurements were also made for Ohmic, L-mode, and $\mathrm{H}$-mode phases of a discharge. The Ohmic and L-mode phases were close to steady state. The pre-transition points (labeled $\mathrm{LH}$ ) were made when a small incremental increase in the neutral beam power (usually 0.3-0.6 MW) caused an L-H transition. The $\mathrm{H}-$ mode points were measured 20-30 ms after the transition. The Thomson scattering measurements are made roughly every $6 \mathrm{~ms}$, and the profiles analyzed are usually the average of 3-4 measurements.

For fixed $I_{P}$ and $B_{T}$, the edge $T_{e}$, evaluated at the $n_{e}$ symmetry point, $T_{e}$ (sym), is typically in the range of 40 to $60 \mathrm{eV}$ and, as shown in Fig. 6, is relatively independent of the edge density. Although the density increases roughly a factor of 2 in $\mathrm{H}$-mode, the temperature remains approximately at its L-mode value. This is likely due to the rapid increase in density after the transition, which may prevent the temperature from increasing. There is little distinction between the Ohmic, L-mode and pre-transition points, indicating $T_{e}$ at this location is insensitive to the heating power. This is expected due to the close proximity of this location to the open field lines in the scrape-off-layer and the associated high parallel heat conduction (For a $1 \mathrm{D}$ heat conduction model on open field lines, $\mathrm{T}_{e}$ depends only weakly on the heating power, varying as $\mathrm{P}^{2 / 7}$ ). It therefore seems unlikely that the temperature at the location where the steep gradients form in $\mathrm{H}$-mode is a key parameter for the $\mathrm{L}-\mathrm{H}$ transition.

In order to facilitate comparisons with other devices, we have evaluated edge parameters $2 \mathrm{~cm}$ inside the separatrix. We find this location roughly corresponds to the edge density knee determined from the hyperbolic tangent fit. Since $T_{e}(\mathrm{sym})$ is relatively constant, this location may serve as a proxy for the edge temperature gradient. An operational space diagram of $T_{e}$ and $\mathrm{n}_{\mathrm{e}}$ evaluated $2 \mathrm{~cm}$ inside the separatrix is shown in Fig. 7. Although there is a trend for pretransition data $(\mathrm{LH})$ to be at higher temperatures, these data are not well separated from the normal L-mode data. Therefore, these parameters do not clearly resolve the $\mathrm{L}-\mathrm{H}$ transition operating space. For comparison, a fit to the LH data on ASDEX-Upgrade [6] given by $\mathrm{T}_{\mathrm{e}}(\mathrm{a}-$ $2 \mathrm{~cm})=145 \mathrm{n}_{\mathrm{e}}^{-0.3} \mathrm{~B}_{\mathrm{T}}^{0.8} \mathrm{I}_{\mathrm{p}}^{0.5}(\mathrm{eV})$ is also shown. The DIII-D data generally fall a factor of 2 below the ASDEX-Upgrade data, indicating that the value of the edge temperature alone is not a critical parameter for the L-H transition. 


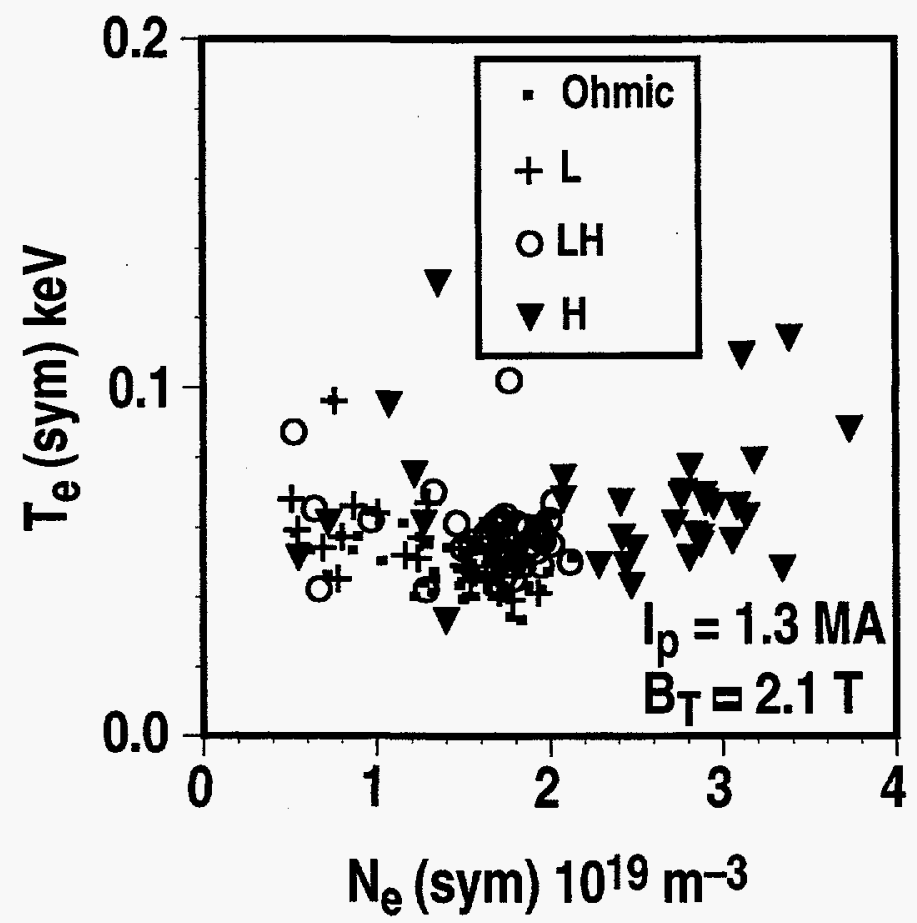

FIG. 6. Operational space diagram for the edge $T_{e}$ and $n_{e}$ evaluated at the $n_{e}$ symmetry point.

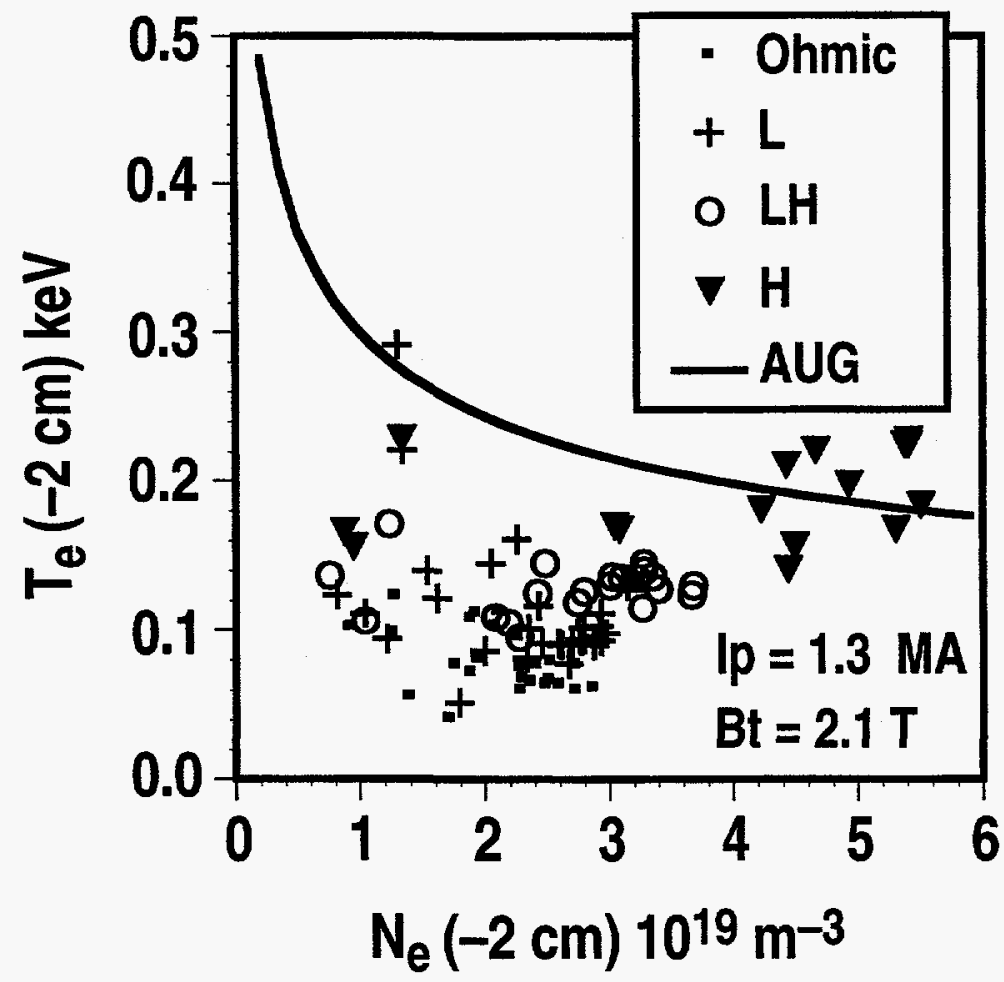

FIG. 7. Operational space diagram for the edge $T_{e}$ and $n_{e}$ evaluated $2 \mathrm{~cm}$ inside the separatrix. A fit to $L H$ data from $A S D E X$-Upgrade is shown for comparison. 


\section{COMPARISON WITH MODELS}

The improved localization of the edge parameters now permits more detailed comparisons with L-H transition theories. In a model based on 3D simulations of the Braginskii equations by Rogers and Drake [7-8], the threshold condition is parameterized in terms of $\alpha_{\mathrm{MHD}}$ and $\alpha_{\mathrm{DIAM}}$, given by:

$$
\begin{aligned}
& \alpha_{M H D}=-R^{2} d \beta / d r \\
& \alpha_{\text {DIAM }}=V_{d i, e^{t_{0}}} / L_{0}
\end{aligned}
$$

where $V_{d i, e}=\rho_{s} c_{s} / L_{p i, e}, \rho_{s}=c_{s} / \Omega_{c i}, c_{s}^{2}=\left(T_{e}+T_{i}\right) / m_{i}, t_{0}=\left(R L_{p} / 2\right)^{1 / 2} / c_{s}, L_{0}=2 \pi q$ $\left(v_{\mathrm{ei}} \mathrm{R} \rho_{\mathrm{s}} / 2 \Omega_{\mathrm{e}}\right)^{1 / 2}\left(2 \mathrm{R} / \mathrm{L}_{\mathrm{p}}\right)^{1 / 4}$. Transport is suppressed when $\alpha_{\mathrm{MHD}}>0.5$ and $\alpha_{\text {DIAM }}>\sim 0.6$.

This model is for shifted circle geomerty which is not representative of the shaped discharges usually run in the DIII-D tokamak. The ballooning parameter for general magnetic geometry is defined as

$$
\alpha_{\mathrm{MHD}}=2 \mu_{0}(\mathrm{dp} / \mathrm{d} \psi) \times(\mathrm{dV} / \mathrm{d} \psi)\left[\mathrm{V} /\left(2 \pi^{2} \mathrm{R}\right)\right]^{1 / 2} / 4 \pi^{2}
$$

where $\mathrm{p}$ is the total plasma pressure, $\psi$ the label for poloidal flux, $\mathrm{V}$ is the volume of a flux surface, $\mathrm{R}$ is the major radius, and $\mu_{0}$ is the permeability constant.

A close approximation for this formula for DIII-D is given by

$$
\alpha_{\mathrm{MHD}} \sim \frac{2 \mu_{0} \mathrm{q}_{95} \sqrt{\mathrm{K}} \operatorname{ar}_{\mathrm{xpt}}^{2}\left(2000 \mathrm{P}_{\mathrm{e}} / \mathrm{L}_{\mathrm{Pe}}\right)}{\mathrm{R}_{\mathrm{o}}\left|\mathrm{B}_{\mathrm{T}}\right|(\mathrm{d} \psi / \mathrm{dR})}
$$

where $\mathrm{a}$ is the minor radius, $\mathrm{r}_{\mathrm{xpt}}$ is the major radius of the $\mathrm{X}$-point, $\mathrm{K}$ is the elongation, $\mathrm{P}_{\mathrm{e}}$ is the electron pressure in $\mathrm{kP}_{\mathrm{a}}$, and $\mathrm{L}_{\mathrm{pe}}$ is the electron pressure gradient scale length.

The operational space of $\alpha_{\text {MHD }}$ and $\alpha_{\text {DIAM }}$, evaluated at the location of the maximun edge density gradient, is shown in Fig. 8 for Ohmic, L-mode, pre-transition LH, and H-mode phases. These parameters are evaluated with the approximations $T_{i}=2 T_{e}, L_{p i}=2 L_{p e}, Z_{e f f}=2$ and $A=2$. The ballooning parameter, $\alpha_{\text {MHD }}$, which contains edge gradients, provides a better separation of the L-mode and pre-transition data than the edge $n_{e}$ and $T_{e}$ in Fig. 7, indicating it may be important for the L-H transition. Due to the lack of separation of the data with $\alpha_{\text {DIAM, }}$, the 
importance of this parameter is not strongly motivated by the data. Further quantitative comparisons will require improvements in the model to include realistic geometry.

In another model of the L-H transition based on the stabilization of Alfvén drift waves by O. Pogutse et al. [9], the threshold condition is parameterized by a normalized beta,

$$
\beta_{\mathrm{n}}=\frac{\beta_{0}}{\mu}=\left(\frac{\mathrm{M}_{\mathrm{i}}}{\mathrm{m}_{\mathrm{e}}}\right)^{1 / 2} \frac{4 \pi \mathrm{n}_{0} \mathrm{~T}_{0 \mathrm{e}}}{\mathrm{B}_{\mathrm{o}}^{2}} \frac{1}{\mathrm{k}_{\|} \mathrm{x}_{0 \mathrm{p}}},
$$

and the normalized collision frequency

$$
v_{\mathrm{n}}=\frac{v}{\sqrt{\mu}}=\left(\frac{\mathrm{M}_{\mathrm{i}}}{\mathrm{m}_{\mathrm{e}}}\right)^{1 / 4} \frac{\mathrm{x}_{0 \mathrm{p}}^{1 / 2}}{\lambda_{\mathrm{e}} \mathrm{k}_{\|}^{1 / 2}} .
$$

Here $B_{0}$ is the toroidal magnetic field, $x_{0 p}=-\left(1 / p_{0} \cdot d p_{0} / d x\right)^{-1}$ characterises the pressure gradient scale length and $\lambda_{\mathrm{e}}$ the mean free path $\left(\lambda_{\mathrm{e}}=\mathrm{V}_{\mathrm{Te}} / \mathrm{v}_{\mathrm{e}}\right)$ and $\mathrm{k} \|$ is the parallel wave number $\left(k_{\|} \sim \mathrm{s} / \mathrm{qR}\right.$, where $\mathrm{s}$ is the shear of the magnetic field $\mathrm{s}=\mathrm{rdq} / \mathrm{qdr}$ and $\mathrm{q}$ the safety factor).

Turbulent transport is suppressed when $\beta_{n}>\beta_{\text {crit }}=1+\nu_{n} / 3$. Fig. 9 shows data evaluated at the maximum edge density gradient on the $\beta_{n}-v_{n}$ plane. The value of $\beta_{n}$ has about the right magnitude but no clear distinction exists between points just before the $\mathrm{L}-\mathrm{H}$ transition and points that remain $\mathrm{L}$-mode or Ohmic. $\mathrm{H}$-mode points, taken just after the $\mathrm{L}-\mathrm{H}$ transition, are well above the threshold condition in both these models. Therefore, comparison of the edge gradients between $\mathrm{L}-$ and $\mathrm{H}$-mode is not particularly useful in distinguishing among these models.

High power thresholds for the L-H transition are observed for the COMPASS-D tokamak at low edge collisionality, $v^{*}<1$. In a model for the L-H transition by Wilson et al. [10], the L-H transition is inhibited by unstable peeling modes at low collisionality. Transport is reduced when $\alpha_{\mathrm{MHD}}>\sim 0.5$ and $v^{*}>\sim 1$. The operational space of $\alpha_{\mathrm{MHD}}$ and $v^{*}$, evaluated at the maximum density gradient, is shown in Fig. 10. Collisionality of the edge plasma varies in the range of 550 , and often increases slightly after the L-H transition as the edge density rises. DIII-D does not operate in the collisionality regime where this model applies suggesting different devices may have different critical parameters for obtaining $\mathrm{H}$-mode. Because of the wide range of collisionality over which $\mathrm{H}$-mode is obtained, collisionality alone is not likely to be a key parameter. However, there is a correlation between $\alpha_{M H D}$ and the value of $v^{*}$ just before the transition. 




FIG. 8. Operational space diagram for the critical parameters of the drift-ballooning instability model of Ref. 8.

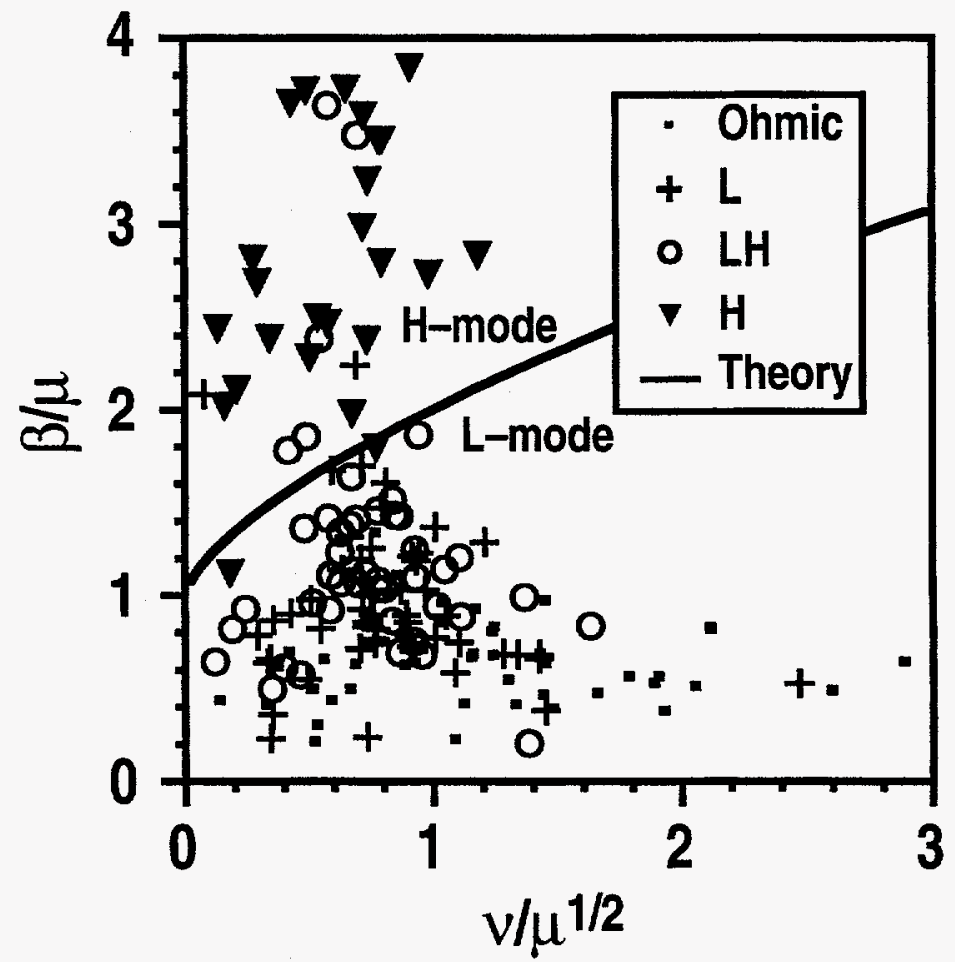

FIG. 9. Operational space diagram for the critical parameters of the Alfvén-drift wave model of Ref. 9. 




FIG. 10. Operational space diagram for the critical parameters of the peeling mode model of Ref. 10. 


\section{VB DRIFT EFFECTS}

The direction of the ion $\nabla \mathrm{B}$ drift relative to the $\mathrm{X}$-point location has a dramatic influence on the magnitude of $\mathrm{H}$-mode power threshold, $\mathrm{P}_{\mathrm{TH}}$. Hinton [11] and later Hinton and Staebler [12] have attributed this effect to neoclassical cross-field fluxes of both heat and particles driven by poloidal temperature gradients on the open field lines in the scrape-off-layer (SOL). The magnitude of these fluxes scale like $\sim(n / r)(T / B)(\partial T / \partial \vartheta)$, where $r$ is the minor radius, $T$ the temperature, and $\vartheta$ the poloidal angle. The flux surface average of these cross-field fluxes is zero unless asymmetries such as the gradient of $\mathrm{B}$ and/or the poloidal temperature gradient lead to a net flux. In its simplest form, these fluxes influence $\mathrm{P}_{\mathrm{TH}}$ by either adding to or subtracting from the power flow to the edge of the plasma. A $1 \mathrm{D}$ analysis of heat conduction in the SOL suggests that these cross-field fluxes can be a significant fraction of the input power if the temperature near the X-point region is sufficiently low [13]. It was proposed that some of the observed scaling of $\mathrm{P}_{\mathrm{TH}}$ is due to the variation of the magnitude of these fluxes and may not be intrinsic to the scaling of the physics of the $\mathrm{L}-\mathrm{H}$ transition itself. For instance, the increase of $\mathrm{P}_{\mathrm{TH}}$ at low density may be due to the reduction of the $\nabla \mathrm{B}$ effect as the sheath limit for parallel heat conduction is reached and the poloidal temperature gradient is reduced. Many qualitative features of this model are in agreement with observations of $\mathrm{P}_{\mathrm{TH}}$ scaling, such as the existence of a density threshold, the importance of the $\mathrm{X}$-point position, and the increase of $\mathrm{P}_{\mathrm{TH}}$ in double-null configurations.

In order to further test these ideas, a series of experiments were carried out in which plasmas with identical operational parameters except for the direction of the toroidal field were compared. In these discharges, the neutral beam power was modulated at a low duty cycle $(12.5 \%$, average power $0.3 \mathrm{MW}$ ) in order to keep the plasma just below $\mathrm{P}_{\mathrm{TH}}$ in the forward $\mathrm{B}$ case $(\nabla \mathrm{B}$ drift toward the $\mathrm{X}$-point). This resulted in power levels far below $\mathrm{P}_{\mathrm{TH}}$ in the reverse $\mathrm{B}$ case where $\mathrm{P}_{\mathrm{TH}} \sim 5 \mathrm{MW}$. Motivated by the idea that edge parameters control the $\mathrm{L}-\mathrm{H}$ transition, we compare the edge $n_{e}, T_{e}, T_{i}$, and $\nabla P_{e}$ profiles evaluated at the knee of the density profile shown in Fig. 11(a-d). There is almost no difference in the value of these parameters between the two directions of the toroidal field, even though one discharge is very near the L-H transition and the other is very far away in terms of power. Also shown in Fig. 11 are the edge parameters for the reverse B case, when the power level is just below the threshold, (5 MW). Although the edge density remains the same, (the line average density was held constant), the edge temperatures and pressure gradients are much greater than in the forward B case.

Preliminary analysis of the divertor conditions show that significant differences between these discharges appear near the X-point region. The electron density just below the X-point 
measured by Thomson scattering in the forward B case is 4-5 times greater than the reverse B case, as shown in Fig. 11(e). The cause of this high-density region and its influence on the L-H transition is under investigation. It may be evidence of the ion $\nabla \mathrm{B}$ drift carrying heat and particles across the $\mathrm{X}$-point into the private flux region, or it may be the result of $\mathrm{E} \times \mathrm{B}$ flows in the divertor.

As previously discussed, several theories of the $\mathrm{L}-\mathrm{H}$ transition consider the edge pressure gradient as a key parameter for the transition (Section 3). As shown in Fig. 11(d), the forward B edge electron pressure gradient is slightly higher than the reverse $\mathrm{B}$ case at $1 \mathrm{MW}$. This may be evidence for cross-field fluxes in the SOL playing a role in determining the edge pressure gradient but the magnitude is too small to have an important effect on the power threshold. Calculations of the cross-field fluxes described above, based on measured SOL and divertor temperatures and densities, result in powers of only a few tens of kilowatts. These fluxes are considered to be too small to contribute significantly to the overall power balance. It is possible, however, that these fluxes affect the edge plasma near the X-point and influence the L-H transition threshold in some other way. For example, modeling of the plasma edge region using UEDGE with the drift terms active [14] has shown changes in the edge $E_{r}$ structure that are consistent with increased $\mathrm{E} \times \mathrm{B}$ shear flow when the ion $\nabla \mathrm{B}$ drift is toward the $\mathrm{X}$-point. 




FIG. 11. Edge parameters of (a) $T_{i}$, (b) $T_{e}$, and (c) $n_{e}$, evaluated at the $n_{e}$ knee location for forward (solid) and reverse $B$ (dashed) $L$-mode discharges where the total heating power was held fixed at approximately $1 \mathrm{MW}$, (just below the $L-H$ power threshold for the forward $B$ case). These parameters, as well as (d) the maximum $\nabla P_{e}$ are nearly identical for both directions of $B$. The electron density just below the $X$-point, shown in panel (e), is significantly greater in the forward $B$ case. The light dashed curves show these parameters for the reverse $B$ case at $5 \mathrm{MW}$, (just below the $L-H$ power threshold for the reverse $B$ case). 







\section{CONCLUSIONS}

A technique of fitting a modified hyperbolic tangent to the edge profiles of $n_{e}, T_{e}$ and $T_{i}$ has eliminated the scatter caused by the flux surface reconstruction and has improved the localization of the plasma edge. Using this technique, we have determined that the position of the maximum edge density gradient remains relatively constant across the L-H transition. Since this is the location where the confinement improvement is greatest, it is a good location to evaluate the local edge conditions relevant to the formation of the edge transport barrier in $\mathrm{H}$-mode.

Near the power threshold, the density increases roughly a factor of 2 in $\mathrm{H}$-mode, but the temperature remains approximately at its L-mode value. Therefore, it seems unlikely that the temperature at the location where the steep gradients form in $\mathrm{H}$-mode is a key parameter for the L-H transition.

Local conditions at the plasma edge are broadly consistent with several models of the L-H transition that use edge gradients in their formulation of a critical threshold parameter. However, the critical parameters in these models do not distinguish between conditions that lead to an L-H transition and those that remain in $\mathrm{L}$-mode.

The ion $\nabla \mathrm{B}$ drift direction has only a small effect on the edge plasma conditions measured near the plasma midplane but a large effect on the divertor plasma. Thus, the dramatic change of the power threshold with the direction of the ion $\nabla B$ drift implies that phenomena in the divertor region may be critical for the L-H transition. Therefore, models of the transition that do not include effects of the field direction are missing important physics for the L-H transition. 


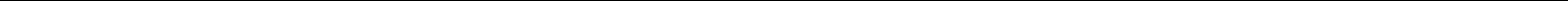




\section{REFERENCES}

[1] CARLSTROM, T.N., GROEBNER, R.J., Phys. Plasma 31867 (1996).

[2] LAO, L.L. et al, Nucl. Fusion 251611 (1985).

[3] CARLSTROM, T.N. et al, Rev. Sci. Instrum. 634901 (1992).

[4] GOHIL, P. et al, Proc. $14^{\text {th }}$ Symposium on Fusion Engineering, San Diego, 1991 (Institute of Electrical and Electronics Engineers, New York, 1992), Vol. 2, p. 1199.

[5] GROEBNER, R.J., CARLSTROM, T.N., Plasma Phys. Control. Fusion, 40673 (1998).

[6] SUTTROP, W. et al., Plasma Phys. Control. Fusion, 392051 (1997).

[7] ROGERS, B.N., DRAKE, J.F., Phys. Rev. Lett, 79229 (1997).

[8] ROGERS, B.N., DRAKE, J.F., ZEILER, A., Proc. $17^{\text {th }}$ IAEA Fusion Energy Conf., Yokohama, Japan 1998, Paper IAEA-CN-69/THP2/01.

[9] POGUTSE, O. et al., "The Alfvén drift-wave instability and the scaling of the edge temperature at the L-H transition," Proc. of 24th EPS Conference, 1997, (P3-1041).

[10] WILSON, H.R. et al, Proc. $17^{\text {th }}$ IAEA Fusion Energy Conf., Yokohama, Japan 1998, Paper IAEA-F1-CN-69/TH3/2.

[11] HINTON, F.L., Nucl. Fusion 251457 (1985).

[12] HINTON, F.L., STAEBLER, G.M., Nucl. Fusion 29405 (1989).

[13] CARLSTROM, T.N. et al., "H-mode threshold power scaling and the $\nabla \mathrm{B}$ drift effect," Proc. of 24th EPS Conference, 1997, (P3-1089).

[14] ROGNLIEN, T.D. et al, to be published in Phys. Plasma. 


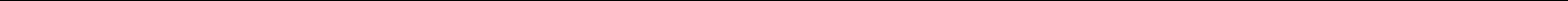




\section{ACKNOWLEDGMENT}

Work supported by the U.S. Department of Energy under Contract No. DE-AC0389ER51114. 